\title{
Lecture(s) et technologies
}

\section{Henri Portine}

\section{OpenEdition}

Journals

Édition électronique

URL : http://journals.openedition.org/ries/4280

DOI : $10.4000 /$ ries.4280

ISSN : 2261-4265

\section{Éditeur}

Centre international d'études pédagogiques

\section{Édition imprimée}

Date de publication : 1 juin 1994

Pagination : 99-110

ISSN : 1254-4590

\section{Référence électronique}

Henri Portine, «Lecture(s) et technologies », Revue internationale d'éducation de Sèvres [En ligne],

02 | 1994, mis en ligne le 17 avril 2015, consulté le 01 mai 2019. URL : http://journals.openedition.org/ ries/4280 ; DOl : 10.4000/ries.4280

Ce document a été généré automatiquement le 1 mai 2019.

(c) Tous droits réservés 


\title{
Lecture(s) et technologies
}

\author{
Henri Portine
}

1 Poser la question du rapport entre l'activité de lecture et les technologies, c'est en fait mélanger deux questions, souvent entremêlées historiquement. La première est celle du support technologique de l'écrit et donc aussi de l'activité de lecture. Ce support contraint la lecture par sa forme et son matériau. Nous allons y revenir. Mais un deuxième type de technologie est à l'œuvre dès lors qu'il s'agit non seulement de lire mais aussi d'apprendre à lire, il s'agit des technologies mobilisées par cet apprentissage.

2 Nous ne chercherons pas ici à élucider l'éventuelle différence entre apprentissage et acquisition. En revanche, il nous semble fondamental de nous interroger sur le correspondant au niveau de l'enseignant de ce qu'est l'apprentissage au niveau de l'apprenant. Ceci nous paraît fondamental parce que notre but n'est pas d'éclairer le processus psychologique de lecture mais d'essayer de mieux comprendre l'apprentissage de la lecture au sein même de la situation pédagogique et donc de la relation enseignantapprenant, et cela - quant au présent article - du point de vue des technologies à l'œuvre.

3 Face à l'apprenant en situation d'apprentissage de la lecture, l'enseignant ne saurait prétendre suivre un algorithme pédagogique puisque nous ne sommes pas (pas encore?) en possession d'une description suffisamment fine de l'apprentissage de la lecture pour prétendre apporter à l'apprenant les éléments dont l'ingestion assurerait le succès de son apprentissage. Dès lors, l'enseignant peut choisir trois conduites :

- proposer à l'apprenant une préparation à la lecture en lui offrant des exercices supposés développer des techniques en jeu dans l'activité de lecture ;

- proposer à l'apprenant des aides à la lecture en lui offrant des supports utilisables parallèlement à ses activités de lecture ;

- proposer à l'apprenant un entraînement à la lecture en lui offrant des activités de lecture modélisantes pour l'activité de lecture visée ou des aides (le dictionnaire, par exemple).

Chacun de ces trois types de conduites pédagogiques peut être exclusif des deux autres. Mais l'enseignant peut aussi chercher à articuler deux d'entre eux, voire les trois. Dans ce cas, il convient bien évidemment de veiller à la cohérence de la démarche d'ensemble du point de vue des activités proposées (activités globalisantes ou analytiques, par exemple). 
5 Nous allons d'abord définir les grandes composantes des technologies supports de l'activité de lecture. Il est important de bien saisir la relativité historique de ces technologies et de nos pratiques traditionnelles. Pour ce faire, il faut reprendre quelques données des temps passés. Cette mise en perspective nous permettra de comprendre la révolution qui est en train de s'opérer sous nos yeux.

\section{Les technologies supports de l'activité de lecture}

Historiquement, l'on peut opposer grosso modo la «lecture continue» et la «lecture discontinue ». La première est liée à l'oralisation (et donc à un transcodage immédiat), la seconde au « travail cognitif » d'un support écrit.

7 La «lecture continue » correspond à deux paramètres: scriptura continua et support déroulant (l'on remarquera que la micro-informatique a renoué avec ce type de support en offrant le «menu déroulant»). Ce support déroulant est le volumen de papyrus puis de parchemin. La fin de la scriptura continua ne coïncide pas totalement avec l'apparition du volumen: sa disparition, plus précoce, annonce l'évolution de la lecture qui produira le codex.

8 La « lecture discontinue » est le corrélat de deux paramètres radicalement différents : le découpage du support en feuilles et sa reliure, et la stabilisation du graphisme. Le découpage et la reliure du support est le codex qui fut peu de temps de papyrus, surtout de parchemin et enfin de papier. La stabilisation (ou nouvelle stabilisation?) du graphisme apparut plus tardivement que le codex, sous Charlemagne d'abord avec la Caroline, puis - surtout - avec l'imprimerie.

9 Dans un premier temps, l'écrit (alphabétique) fonctionne comme une transcription de l'oral et donc en écriture continue, sans blancs séparateurs; c'est la scriptura continua. Mais cette notation ne facilite pas l'intelligibilité de l'écrit. Pour être maximale, cette intelligibilité suppose un équilibre entre division et globalité : des « mots » composés trop longs gênent cette intelligibilité, mais des « mots » trop courts (imaginons que l'on sépare certains suffixes de leur base) ont la même conséquence. La scriptura continua devait donc se diviser en unités graphiques, les mots. Cependant cette division, bien qu'elle rompe la continuité de l'écriture, ne rompt pas totalement la continuité de la lecture, elle la rend seulement plus aisée. Ce sera le passage du volumen au codex qui assurera véritablement le passage à la « lecture discontinue ».

10 Avant même le volumen, le premier support connu est sans doute la tablette dont l'ardoise est en quelque sorte un lointain descendant (outil de l'enseignement mutuel, fin XVIII et $\mathrm{XIX}^{\mathrm{e}}$ siècles). Mais le support tablette est lié à la rigidité du matériau. Constituer un support flexible (et donc aisément stockable) de grande dimension et pour un usage collectif (au sens que peut prendre ce mot dans l'Antiquité) suppose une technologie de fabrication assez avancée. Ce sera le volumen, rouleau qui se lit (ou se déchiffre) du début à la fin et de façon continue. C'est là le deuxième paramètre de la « lecture continue ».

11 Le volumen, rouleau en moyenne de six à dix mètres de feuilles mises bout à bout est support de la mémoire, il permet le discours (l'art oratoire ou rhétorique). Il induit une lecture continue, marquée par l'oralisation et une conception linéaire : la lecture va du début à la fin du document. La reprise d'un élément d'une page qui précède, le fait de feuilleter, de mettre en rapport, supposeront un support plus aisément consultable. 
12 Aux deux conditions énoncées plus haut (remplacement du volumen par le codex et stabilisation du graphisme de l'écrit) vont s'en ajouter deux autres pour assurer un caractère dominant à ce que nous avons nommé plus haut « lecture discontinue » :

- la désacralisation de l'écrit lorsque l'écrit était conçu directement en rapport au sacré : quand l'écrit est lié au religieux il doit répondre à des critères esthétiques qui nuisent à la reproductibilité de l'image des mots, le poids culturel du mot est plus fort et sa lisibilité est donc réservée aux « lettrés »; de plus, un écrit lié au religieux demande un respect littéral, or la littéralité dans l'activité de lecture transforme celle-ci en exégèse, difficulté supplémentaire ;

- la socialisation de l'écrit : en se répandant l'écrit devient utile et cet utilitarisme va jouer un rôle double, l'écrit utile doit être simple d'accès, mais aussi l'utilité motive l'apprentilecteur.

13 Bien évidemment, il ne faut pas voir dans ce qui précède une description historiquement ordonnée. La chronologie est plus sinueuse. Tous les critères n'apparaissent pas en même temps. Et il peut même se présenter des retours en arrière.

Le codex fait place peu à peu à la "saillance" de certains éléments (le transcodage oralisant rendait inutile toute saillance). La lecture peut devenir discontinue. Plusieurs siècles plus tard, elle va pouvoir être de deux types : la «lecture suivie » et la «lecture consultation ». La « lecture suivie » est celle des romans mais aussi des ouvrages qu'il faut lire. La "lecture consultation» est celle du journal mais aussi de la brochure professionnelle dans laquelle il faut trouver certaines informations. La «lecture suivie » est donc une lecture tournée vers le plaisir ou une lecture dans laquelle l'appréhension de phénomènes argumentatifs (toujours complexes) prend une grande importance. La « lecture consultation » est une lecture qui limite le langage à sa valeur informative.

Historiquement, le livre est principalement soit un objet de savoir (culturel ou "scientifique ", d'où la possibilité des deux types de lecture discontinue), soit un objet esthétique destiné à parer la bibliothèque d'un noble riche puis d'un riche bourgeois. Mais ce n'est que tardivement que la "lecture consultation» fera massivement son apparition, avec le développement des journaux d'abord, avec la notion d'écrit professionnel (et non plus d'écrit savant) ensuite.

Le développement de l'écrit public (affiches, panneaux de signalisation urbains, journaux aux différents formats) a entraîné une légère augmentation du nombre des supports. Tous ces supports correspondent à une « lecture consultation ».

Mais l'accroissement véritable est en train de se produire : lecture sur écran de microordinateur, lecture d'un journal papier, d'un journal mural, d'un journal vidéo, d'une affiche, d'une télécopie, d'un livre, d'une microfiche, d'une page de télétex (appelé Minitel en France), autant de mises en œuvre différentes de l'activité de lecture.

\section{La disparition du livre?}

18 L'apparition de l'électronique et de l'informatique a fait naître l'idée que le livre était condamné à disparaître. Cette idée a pris deux formes : le remplacement du texte et donc du livre par l'image (Mac Luhan) et le remplacement du livre par le support informatique, avec dans ce cas conservation du texte. 
19 Les prédictions de Mac Luhan sur l'ère électronique de l'image remplaçant l'ère de l'écrit et du livre ne semblent pas se réaliser (en tout cas pas aussi vite que prévu) et la plupart de ceux qui avaient enfourché la nouvelle mode ont déjà fait amende honorable (mais la toute dernière mode ne demande-t-elle pas que l'on se rassemble dans une grande salle sous l'œil attentif et sévère du professeur Bernard Pivot?). Le livre perdure mais peutêtre pas à la façon dont on entend l'écho des sentences des éternels gourous virevoltants. Ce n'est pas la permanence du livre qui s'annonce, c'est la coexistence de médias différents sans qu'il y ait forcément de médium dominant. Et là est peut-être la véritable nouveauté. Les nouvelles technologies n'excluent pas les anciennes. Dans les bibliothèques, on réalise des microfiches pour économiser de la place et en même temps on prône la mise en contact du public avec les ouvrages imprimés, ce qui «perd» de la place. Mais il n'y a pas contradiction pour autant. La microfiche est plutôt réservée à l'ouvrage de référence, le livre en rayon s'apparente à l'étalage des supermarchés: le plaisir de la découverte.

20 Le remplacement systématique du livre par un support informatique ne semble guère plus crédible pour plusieurs raisons. La première est liée aux fantasmes producteurs de cette idée de remplacement systématique. On en trouve une remarquable illustration dans un article récent consacré par F. Gruhier à la "Bibliothèque de France ${ }^{1}$ ». Ces fantasmes sont assez largement partagés, c'est pourquoi ils méritent au moins une mise en garde. "On allait enfin être débarrassé de ces tonnes de fragiles et encombrants « codex » de papier [...] menacés par l'humidité, la lumière, les insectes, les incendies, les vandales, les voleurs. » Suggérons à l'auteur de l'article de placer un $\mathrm{CD} \mathrm{ROM}^{2}$ dans un lieu humide, ou de le soumettre à un incendie. Il verra le résultat dans les deux cas. Quant aux insectes, ils n'apparaissent que dans des stockages défectueux (l'informatique a aussi ses exigences), ou sont le résultat d'une longue conservation (que sait-on du vieillissement des stockages informatisés ?) : et les vandales? Les voleurs? L'on pourrait d'ailleurs poursuivre le parallèle: les ouvrages du XIX siècle demandent que l'on apprenne à les lire; les disquettes établies en 1983 (il y a dix ans !) ne sont plus lisibles sous DOS 5 ou $6^{3}$, il faut soit les transcoder continuellement, soit conserver les différentes étapes de lecture (système d'exploitation et logiciel de SGBD ${ }^{4}$ ou de traitement de texte).

21 La seconde raison est purement expérimentale. On sait depuis de nombreuses années stocker sur microformes (microfiches et microfilms). Lorsque l'on dépose une thèse, l'Atelier national de reproduction vous en fournit plusieurs exemplaires sous forme de microfiches. Essayez de distribuer ces microfiches autour de vous. Seules les bibliothèques les acceptent, ce qui prouve que le gain de place n'est pas le seul argument en cause. D'ailleurs qui est prêt à remplacer sa bibliothèque professionnelle (et ne parlons pas de la bibliothèque du salon) par un ou deux tiroirs de microfiches?

La troisième raison est liée à la lecture. D'abord, la lecture est plurielle et ne s'accommode pas dans son ensemble des procédures de consultation informatique. Ensuite, l'inventivité, liée aux hasards de rencontres dans le feuilletage d'un livre par exemple, disparaîtrait complètement dans une bibliothèque n'utilisant que des supports informatiques. Les algorithmes de consultation ne connaissent pas le chaos de la pensée, le hasard de la rencontre de deux idées qui en produisent une nouvelle. 


\section{Technologies actuelles et lecture} discontinue suivie" n'est ni une contradiction dans les termes, ni un oxymore. Actuellement, toute lecture est discontinue parce que plus personne n'est censé oraliser pour comprendre. La lecture continue est une lecture en deux étapes : 1) oralisation, 2) compréhension. Elle correspond parfaitement à la technologie du volumen même si elle a été encore utilisée pendant de nombreux siècles avec le codex. Toute lecture silencieuse est discontinue (mouvements de l'œil, mais aussi mouvements de la pensée pour vérification ou recherche d'un complément). Sa technologie de base est celle du codex qui permet le va-et-vient. Or cette lecture discontinue peut être en même temps suivie. L'exemple type en est la lecture d'un roman policier. Le lecteur revient en arrière pour bien apprécier la description du lieu du meurtre, pour vérifier qu'Angèle est bien la cousine et donc la seule descendante d'Auguste susceptible d'en être l'héritier. Mais cette lecture est aussi suivie: on ne lit pas la fin en premier, on cherche à apprécier un crescendo. On voit mal comment le livre, donc le codex, ne demeurerait pas le support de cette lecture. Cela s'applique aussi à la lecture-plaisir en général et à la compréhension de la pensée d'un philosophe par exemple. Le toucher jouera certainement un rôle dans cette conservation, car si le livre peut être objet de cauchemar pour certains conservateurs de bibliothèques (mais ne généralisons pas abusivement), il peut être objetcompagnon pour le lecteur. Cela est plus difficile avec le CD ROM.

Par opposition à la «lecture discontinue suivie», on trouve la «lecture discontinue consultation». Celle-ci peut être de deux types, nous l'avons vu: une "consultationdécouverte » et une " consultation-recherche ». Pour la «consultation-découverte», les choses sont moins tranchées que pour la «lecture suivie». Elle peut se faire sur microfiches et peut-être développera-t-on un jour des lecteurs de microfiches moins encombrants que ceux qui existent actuellement. Elle peut encore être faite à partir du livre : il est plus facile de découvrir une idée nouvelle en furetant sur un rayonnage que dans un tiroir. Elle pourra certainement se faire aussi à partir d'une lecture informatisée. Mais il faut bien voir que le passage du livre à la microfiche puis au CD ROM suppose une prédétermination de plus en plus précise de la source de la découverte : nous avons déjà évoqué les tiroirs des microfiches ; le CD ROM - disque compact que l'on peut lire sans pouvoir le modifier - ne permet pas de fureter!

La lecture « consultation-recherche » est le domaine privilégié de la lecture informatisée. Certes les index existent déjà dans les livres. Mais la puissance de l'outil informatique donne une grande souplesse à la recherche. Localiser tel mot dans une base de données est très rapide. Le plus long et le plus coûteux est de constituer les bases de données en question et le plus difficile est de les structurer de façon adéquate par rapport aux attentes et aux besoins des futurs utilisateurs.

27 Quelles sont les forces et les faiblesses de la « lecture informatisée » ? Elle est très rapide dès lors que l'on sait ce que l'on cherche et que cette recherche a été prévue dans la

Revue internationale d'éducation de Sèvres, 02 | 2015 
programmation de la circulation au sein des documents. C'est là une force, mais c'est aussi une faiblesse. La lecture s'apparente à celle du volumen : elle est liée au défilement. C'est là une deuxième faiblesse. Elle est due à la notion de fenêtre : le texte est considéré comme défilant sous une fenêtre, l'écran. On pourrait atténuer cette faiblesse par un défilement par pages à l'aide d'écrans assez grands et des touches portant une flèche empennée vers le haut ou vers le bas, présentes sur les claviers des PC mais absentes sur les claviers des Macintosh, mais remarquons à ce propos que ce qui se développe actuellement, c'est plutôt l'ascenseur en marge droite de l'écran qui correspond bien à la lecture continue du volumen. Deuxième force, elle permet un très grand stockage sous un volume très réduit et assez fiable, le CD ROM (les autres supports, disquettes et disque dur, sont beaucoup moins fiables). Ce support est idéal pour les bases de données: horaires de trains, fichiers d'adresses, recueils de citations, collections d'images.

On retrouve ici une question déjà traitée : le livre disparaîtra-t-il ? Peut-être un jour. Mais il faudrait que toutes les lectures autres que la lecture "consultation-recherche" meurent ou deviennent très minoritaires. Pourrait-on alors vraiment parler de circulation du savoir? Le livre résistera au moins sur plusieurs générations. Que l'on songe aux siècles qu'il a fallu pour passer de la lecture continue à la lecture discontinue malgré l'invention du codex. Il est donc inutile de tabler sur cette disparition sauf si l'on veut faire rêver (un monde de la légèreté et de l'abstraction) ou faire peur (un monde de robots).

À quelle modification du paysage technologique assiste-t-on à l'heure actuelle ? Comment l'activité de lecture va-t-elle évoluer dans les prochaines années?

On va sans doute vers la multiplicité des supports selon le type de lecture mais aussi selon le degré de conservation de l'écrit. Ainsi, la télécopie est-elle un intermédiaire entre le volumen et le codex. C'est une séquence (comme pour le volumen) de feuillets (comme pour le codex) et ces feuilles volantes ne sont pas sans rappeler la réaction négative des parents quand ils découvrirent roulés en boule au fond du cartable les feuillets ronéotés donnés par des instituteurs soucieux de la participation active de leurs enfants. C'est donc un support archivable. Sans archivage, il disparait ou devient inutilisable. Nous avons donc deux paramètres dominants: le type de lecture et la finalité du document. Ces deux paramètres sont liés bien entendu.

31 À cet éclatement des supports de lecture correspond l'éclatement des technologies médiatrices, c'est-à-dire permettant d'apprendre à lire.

\section{Les technologies médiatrices de préparation à la lecture}

Tant que l'écrit a été considéré principalement comme une transcription de l'oral - et cela a bien dépassé le stade pourtant corrélé du volumen -, la préparation à l'activité de lecture n'a été qu'une préparation à l'oralisation des graphismes (dont on peut désigner les unités par graphème).

33 Apprendre à regrouper les mots par séquences (les " empans »). Il s'agit sans doute des premiers exercices réalisés sur micro-ordinateur. On propose à l'élève trois types d'exercices : englober d'un coup d'œil un groupe de mots (en général à l'aide d'un point pour la fixation de l'œil) ; discriminer des parties de mots dans une liste ; élargir l'empan en le faisant varier. Ces exercices sont inspirés de la lecture rapide, notamment de la 
version élaborée par F. Richaudeau. On les trouve d'abord dans la première version d'ELMO de l'AFL et dans sa forme papier, ATEL 5 .

Se forger une conception de la cohérence des textes. On peut regrouper deux types de pratiques opérant à ce niveau. Le premier est celui des exercices du type «chassez l'intrus ", il faut trouver ou ignorer (dans le cas où l'on réécrit) les éléments proposés non cohérents avec l'ensemble du texte. Le second type se trouve aussi dans ELMO : poser des questions (d'où une conception de la lecture comme recueil d'informations) dont la réponse est facilitée par la perception d'une cohérence au sein de l'ensemble du texte.

Apprendre à gérer la longueur. Le problème est bien connu: comment passer de fragments à du texte. Ce problème se rencontre dans les premiers apprentissages lorsqu'ils sont de type syllabique, il faut passer des mots aux phrases et aux courts textes. Ce problème se rencontre aussi en langue maternelle entre le CM1 et la sixième : passer de courts textes scolaires aux longs textes des bibliothèques de différentes couleurs. Il faut aussi faire face à ce problème en langue étrangère, surtout dans les apprentissages nommés «fonctionnels » (professionnels). Le CREDIF ${ }^{6}$ a publié un dossier sur cette question dans le domaine des sciences économiques et sociales.

\section{Les technologies médiatrices d'aide à la lecture}

Apprendre à élucider le sens d'un mot. Le réflexe est connu : le novice s'arrête aux mots qu'il ne comprend pas, parfois aux mots qu'il ne sait pas prononcer. Cela lui fait perdre le fil de la compréhension. En langue étrangère, le problème est légèrement différent : le débutant tend à s'accrocher à la littéralité des mots. Lorsqu'il ne les comprend pas, il est arrêté lui aussi. Mais même lorsqu'il les comprend, sa compréhension générale du sens en souffre. On peut tenter d'effacer ou d'au moins diminuer ce réflexe chez le lecteur novice (exercices de déduction de sens à partir du contexte, exercices de globalisation du sens). Mais on peut aussi décider d'agir à ce niveau. L'hypertexte est ici mobilisable: on programme sous forme de boutons (cf. ci-dessous) les mots difficiles, l'activation du bouton ouvrant une fenêtre sur l'écran et donnant le sens du mot. On peut aussi proposer au lecteur deux sens différents avec - éventuellement - une analyse de réponse.

Rechercher des contextes différents ${ }^{7}$. Le lecteur comprenant mal l'emploi d'un mot (dont il connaît déjà au moins l'un des sens) dans un texte donné ou ne comprenant pas un mot qui joue un rôle clé dans un texte, recourt à une procédure automatique qui lui donne à voir différents emplois de ces mots et leur(s) contexte(s) en puisant dans une base de données. Cela permet un travail d'élucidation de sens à partir des environnements.

Se faire (et apprendre à se faire) une image du texte, non pas une image graphique, mais une image conceptuelle. Le logiciel permet une analyse et une présentation du texte donnant les regroupements lexicaux (d'où une vision « géographique » de la construction du sens), la liste des fréquences (appréhension plus globale), etc. Il s'agit d'apprendre à voir un texte en lui associant un certain « relief sémantique ».

\section{Les technologies médiatrices d'entraînement à la lecture}

S'entraîner à la formulation d'hypothèses. La technologie, un peu rudimentaire, du rétroprojecteur permettait de masquer des parties du texte d'où la possibilité de 
demander aux apprenants de formuler des hypothèses, puis de découvrir les parties masquées et de demander si les hypothèses proposées se trouvent infirmées ou confirmées avec justifications à l'appui. On peut partiellement automatiser ces procédures.

S'entraîner à la reformulation. Proposer des formulations différentes, des résumés de parties du texte permet de s'entraîner à la lecture dans la mesure où le lecteur doit opérer de temps en temps des synthèses partielles pour avancer dans sa lecture. Ces exercices peuvent se réaliser dans la classe, éventuellement d'ailleurs sous forme d'atelier d'écriture (ou plutôt dans ce cas de réécriture). Ce travail peut se faire aussi bien pour la lecture en langue étrangère que pour la lecture en langue maternelle. Cependant, la lecture en langue étrangère demande un travail plus approfondi à ce niveau, dans la mesure où - comme nous l'avons déjà noté- la littéralité du message freine plus le lecteur non natif que le lecteur natif. Ce type d'exercices peut aussi être automatisé surtout depuis que l'augmentation de la capacité mémoire en mémoire vive permet des partages d'écran.

41 Apprendre à définir des regroupements (des groupes formant un sens). Ces groupes peuvent être de deux types, syntaxique (intra-phrastique), sémantique (transphrastique). Le premier type travaille sur une micro-compréhension, le second sur une macrocompréhension. Ce type d'exercices est difficilement automatisable dans le contexte actuel. Il suppose un agent humain vérificateur, et donc un enseignant. On peut toutefois l'automatiser si un enseignant en assure la correction (ou plutôt peut-être, dans ce cas, la remédiation).

Écrire pour lire. La compréhension dans l'acte lexique mobilise des opérations de reconstruction. On l'a vu dans le cas des reformulations. On peut tenter de prendre le problème à un niveau plus général. On l'a déjà fait en faisant un atelier d'écriture de nouvelles pour apprendre à lire des romans, par exemple (appropriation des modes de construction narrative). ELMO 2000 contient un module de genèse de texte (on peut toutefois se demander s'il s'agit d'écrire pour lire ou de se plier au fait que l'activité dominante à l'école est celle d'écriture). Le problème est alors de ne pas perdre de vue le but : à trop se plier aux processus rédactionnels, on fait de l'écriture, plus de la lecture.

\section{Les utilisations des technologies informatisées}

\section{Les stockages en mémoire et les bases de données}

Les stockages sur disquette ou sur disque dur sont très fragiles, c'est pourquoi il fallait trouver un support plus fiable, ce fut le CD ROM, disque compact porteur de données. Mais comme son nom l'indique ( $\mathrm{ROM} »$ : read only memory), le CD ROM ne peut être que lu. Il est donc impossible de l'annoter. De grande capacité, il permet de stocker des bases de données importantes. Les premières bases de données étaient conçues sur le modèle du fichier d'adresses. On possède de plus en plus des bases de données textuelles et des bases de données structurant de façon complexe différente types d'objets. Une base de données textuelle suppose un programme spécifique permettant d'en extraire les informations recherchées (par exemple: les contextes dans lesquels apparaît un mot donné). 


\section{Les didacticiels}

44 Ce sont des programmes proposant des exercices. Ils supposent résolues deux grandes difficultés. La première est de faire travailler ensemble et surtout de faire s'entendre des informaticiens et des pédagogues (chacun ayant une vue plus ou moins fausse des travaux et des potentialités de l'autre). La seconde est l'analyse des réponses erronées que l'on s'attend à recueillir dans les réalisations de l'exercice. Cela est très difficile pour trois raisons: il faut s'attendre aux erreurs qui se produiront effectivement; il faut comprendre d'où proviendront ces erreurs ; il faut proposer des commentaires qui seront à la fois compréhensibles par l'utilisateur et bénéfiques pour lui. L'activité de lecture étant très polymorphe, l'on sait peu recourir aux didacticiels.

\section{Les langages-auteurs}

Un didacticiel est prédéterminé. Il semble donc opportun de proposer des didacticiels modifiables par les enseignants ou par les responsables de formation, lesquels ne sont pas forcément de fins stratèges de la programmation structurée (ou autre). On obtient alors les langages auteurs: des langages de programmation très orientés vers un certain type de produits et utilisables par tout un chacun possédant (toutefois) simplement un solide bon sens logique. On peut alors choisir ses textes et les faire travailler par l'apprentilecteur.

\section{L'hypertexte}

Il s'agit d'un texte dont certains éléments (nommés «boutons»), des mots en général, peuvent être activés par un clic de souris par exemple. Cette activation ouvre une fenêtre sur l'écran qui fournit des informations. Ce peut être un autre contexte du mot considéré, une explication de ce mot, une donnée supplémentaire (par exemple, la date d'un événement historique).

\section{L'intégration multimédia}

47 Depuis quelques années, on sait intégrer des outils multimédias alors qu'auparavant on juxtaposait ces outils. Intégrer des outils multimédias, cela signifie construire un programme unique gérant ces différents outils (actuellement: son provenant d'un système hi-fi ou d'un synthétiseur, texte, images scannérisées, images d'un magnétoscope - le son et l'image étant de plus en plus obtenus par numérisation). Le langage auteur le plus connu actuellement pour cette intégration multimédias est sans doute authorware, fonctionnant sous standard Macintosh, mais le concept d'intégration multimédias est aujourd'hui en plein développement. On peut utiliser de l'image et du son pour accompagner la compréhension d'un texte. Mais il s'agit encore de procédures lourdes.

\section{Des technologies « avancées » pour la lecture?}

Les didacticiels d'EAO 8 laissent insatisfaites de nombreuses personnes du fait du caractère très limité des procédures envisageables. C'est pourquoi l'on a, vers 1978-1988, fondé 
certains espoirs sur l'EIAO (I pour " intelligent») qui devait recourir à l'architecture des systèmes experts : une base de données, un moteur d'inférences contenant des règles permettant de raisonner sur les éléments de la base de données et un programme gérant le tout et assurant l'interface avec l'utilisateur. Les systèmes experts avaient alors donné certaines preuves de leur intérêt notamment en géologie et en médecine (aide au diagnostic).

Peut-on imaginer une procédure d'aide à la lecture par système expert? Sans aucun doute. Mais dans ce cas, il ne s'agirait plus d'apprentissage de la lecture mais d'une activité professionnelle de type documentaire. On sait que le $\mathrm{XXI}^{\mathrm{e}}$ siècle sera confronté à deux grands problèmes, d'ailleurs tous deux plus ou moins liés à la lecture: la documentation (la masse grandissante des informations rend celle-ci confuse et peu exploitable) et la formation (comment former à des activités de plus en plus " pointues » et dans des contextes de plus en plus mouvants?). Il est possible d'imaginer des systèmes experts d'aide à la synthèse et donc "guides de lecture " dans le domaine de la documentation qui serait alors non pas une documentation informatisée mais une documentation assistée par ordinateur.

Plus lourds et donc peu rentables, on peut envisager des systèmes experts d'apprentissage de la lecture fonctionnant sur le calcul des hypothèses formulables à tel niveau de la lecture. Ainsi, l'apprenti-lecteur se verrait-il demander de formuler une hypothèse à la suite de laquelle le moteur d'inférences du système expert établirait un calcul de probabilité de la confirmation ou de l'infirmation de cette hypothèse à partir des éléments disponibles pour l'utilisateur. Mais on se trouve ici à peu près dans la situation de celui qui utiliserait un bulldozer pour déplacer un pot de fleurs. C'est d'ailleurs toujours un peu le cas - au moins actuellement - en EIAO. Mais peut-être faut-il en passer par là pour aller de l'avant.

Il est important de ne pas prendre l'outil pour l'objectif. La justification du marteau c'est de planter des clous, ce n'est pas l'esthétique du marteau lui-même. Les technologies avancées peuvent être très utiles dès lors que l'on sait les utiliser à bon escient. Mais il ne faut jamais oublier que la question technologique est une question qui suppose toujours une mise en perspective historique et conceptuelle.

\section{NOTES}

1. Le Nouvel Observateur, 30 septembre 1993.

2. CD ROM : disque compact porteur de données consultable sur ordinateur.

3. DOS : Disk Operating System (type de fonctionnement informatique, par opposition au système Macintosh).

4. SGBD : système de gestion des bases de données.

5. ELMO : logiciel proposant à l'enseignant les outils nécessaires pour mettre en place un atelier de lecture.

AFL : Association française pour la lecture.

ATEL : Ateliers de la lecture sur papier conçus pour un public scolaire de 8 à 12 ans. 
6. CREDIF : Centre de recherche et d'étude pour la diffusion du français.

7. Cette idée est à la base du Lecticiel de D. Lehman et A. Pelfrêne (CREDIF), de même que la procédure d'aide-visualisation présentée ci-dessous.

8. EAO : enseignement assisté par ordinateur ; en anglais : CAL : computer assisted learning.

\section{RÉSUMÉS}

L'auteur rappelle d'abord quels furent les supports de lecture de l'Antiquité à nos jours, du codex à l'ordinateur. Il montre que les modalités d'accès au texte évoluent en fonction de ces divers supports.

INDEX

Mots-clés : lecture, histoire de l'écriture

\section{AUTEUR}

\section{HENRI PORTINE}

Université Charles-de-Gaulle, Lille III 